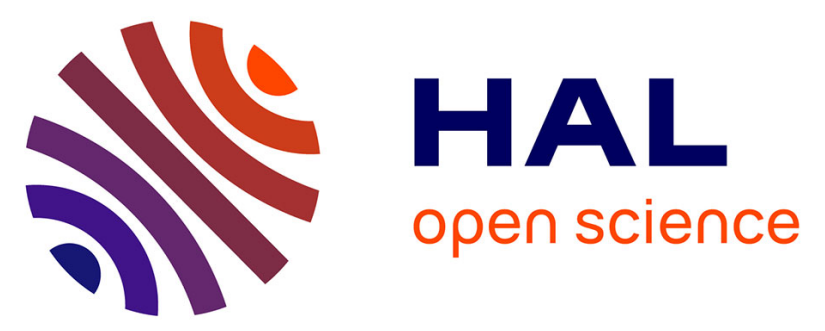

\title{
Traces d'impacts de projectiles sur le gibier : résultats comparés des tirs expérimentaux à l'arc et au propulseur effectués au CEDARC (Treignes, Belgique) en 2003 et 2004.
}

Jean-Marc Pétillon, Claire Letourneux

\section{To cite this version:}

Jean-Marc Pétillon, Claire Letourneux. Traces d'impacts de projectiles sur le gibier : résultats comparés des tirs expérimentaux à l'arc et au propulseur effectués au CEDARC (Treignes, Belgique) en 2003 et 2004.. Annales de Paléontologie, 2008, 94 (3), pp.209-225. 10.1016/j.annpal.2008.05.001 . halshs-00391834

\section{HAL Id: halshs-00391834 \\ https://shs.hal.science/halshs-00391834}

Submitted on 5 Jun 2009

HAL is a multi-disciplinary open access archive for the deposit and dissemination of scientific research documents, whether they are published or not. The documents may come from teaching and research institutions in France or abroad, or from public or private research centers.
L'archive ouverte pluridisciplinaire HAL, est destinée au dépôt et à la diffusion de documents scientifiques de niveau recherche, publiés ou non, émanant des établissements d'enseignement et de recherche français ou étrangers, des laboratoires publics ou privés. 


\title{
Traces d'impacts de projectiles sur le gibier : résultats comparés des tirs expérimentaux à l'arc et au propulseur effectués au CEDARC (Treignes, Belgique) en 2003 et 2004.
}

\author{
Jean-Marc Pétillon \\ Laboratoire Archéologies et sciences de l'Antiquité (UMR 7041) \\ Département d'ethnologie préhistorique \\ Maison René Ginouvès \\ 21 allée de l'Université \\ F-92023 Nanterre cedex \\ jean-marc.petillon@mae.u-paris10.fr \\ Claire Letourneux \\ Max-Planck Institute for Evolutionary Anthropology \\ Department of Human Evolution \\ Deutscher Platz 6 \\ D-04103 Leipzig \\ claire.letourneux@eva.mpg.de
}

\section{Résumé}

Deux séances de tir expérimental à l'arc et au propulseur co-organisées avec P. Cattelain eurent lieu au CEDARC / Musée du Malgré-Tout (Treignes, Belgique) en 2003 et 2004. Leur principal objectif était de reconstituer le fonctionnement des armatures de projectile en bois de cervidé du Magdalénien supérieur d'Isturitz. Le projet incluait également l'étude des stigmates d'impact sur les ossements des quatre animaux-cibles utilisés, étude qui fait l'objet de cet article. L'observation des 127 traces nous a incité à réviser en partie la nomenclature de P. Morel. Par ailleurs, nous avons relevé certaines corrélations entre la nature de la trace d'impact, la morphologie et le degré de minéralisation de l'os. En revanche, nos observations ne nous ont pas permis de distinguer l'utilisation de l'arc de celle du propulseur.

Mots-clés : expérimentation, arc, propulseur, traces d'impacts sur os.

\section{Title}

Projectile impact traces on game: comparative results of the bow and spearthrower experiments held at the CEDARC (Treignes, Belgium) in 2003 and 2004

\begin{abstract}
In 2003 and 2004, two series of projectile experiments were organized by P. Cattelain and ourselves at the CEDARC / Musée du Malgré-Tout (Treignes, Belgium). The experiments involved the use of two male ox calves and two female fallow deers as targets for bow and spearthrower shooting. Our main focus was a functional analysis of the antler projectile tips from the Upper Magdalenian layer of the Isturitz cave site (Pyrénées-Atlantiques, France). The study of the impact traces on the bones of the target animals was also included in the project; its results are presented here. The observation of these 127 traces led us to reconsider the nomenclature suggested by P. Morel, and to distinguish between 3 major traces: notches, punctures and perforations. Correlations appear between the nature of the trace and the shape of the bone (e.g., scapulae strongly associated to perforations), and the bone's mineral content: compared to adult long bones, immature long bones are much more easily perforated by
\end{abstract}


projectile points, which usually remain firmly caught in them. However, our results do not show a clear distinction between the impact traces left by the bow and by the spearthower.

Keywords : experimentation, bow, spearthrower, impact traces on bone.

Par définition, l'histoire taphonomique d'un ensemble osseux commence avec le processus qui déclenche le passage de la biocénose à la thanatocénose. L'étude des causes de la mort d'un animal et, entre autres, des traces que celles-ci ont pu laisser sur ses ossements, fait dès lors partie intégrante de la démarche taphonomique. Et l'action de l'homme, en tant qu'agent taphonomique, figure bien sûr parmi ces possibles causes de décès. C'est dans cette perspective que nous présentons ici ce texte consacré à la caractérisation des traces que laissent les armes de chasse - plus particulièrement les pointes de projectile en matières osseuses - sur le squelette du gibier.

En 2003 et 2004, dans le cadre de la thèse de l'un d'entre nous (J.M.P.), nous avons coorganisé avec P. Cattelain deux séances de tirs expérimentaux qui se sont déroulées au CEDARC / Musée du Malgré-Tout (Treignes, Belgique). Leur objectif principal était de constituer un référentiel de stigmates d'utilisation pour interpréter les fractures des pointes de projectile en bois de renne du Magdalénien supérieur de la grotte d'Isturitz (résultats publiés in : Pétillon, 2005, 2006). Lors de ces expérimentations, nous avons également mis en place un volet archéozoologique consacré à l'étude des traces d'impacts de projectiles sur les ossements des animaux-cibles. Ce type de données n'a jusqu'ici fait l'objet que de peu de publications détaillées, à l'exception notable des travaux de P. Morel $(1993,2000)$ et d'U. Stodiek (1991, p. 254-255 ; 1993, p. 206 ; 2000, p. 76-77). Nous souhaitons ici exposer nos résultats en proposant une synthèse comparative des deux expérimentations, 2003 et 2004. Les résultats de 2003 ont fait l'objet d'une présentation préliminaire (Pétillon et Letourneux, 2003-2004).

Notre objectif était de construire un référentiel de traces d'impacts de projectiles, afin de permettre une meilleure reconnaissance de ce type de stigmates sur les vestiges de faune en contexte archéologique. Pour cela, nous avons chercher à caractériser ces traces d'impacts en explorant les facteurs à l'origine de leur formation. A priori, on pouvait en effet penser que la morphologie de ces traces pouvait être influencée par deux types de facteurs :

- les facteurs intrinsèques à l'animal touché : sa physionomie (plus ou moins robuste ou gracile), son âge (et donc le degré d'ossification des os), la morphologie de l'os (plat, long, court) et sa structure (densité notamment);

- les facteurs extrinsèques : le type d'arme utilisé, les dimensions et la masse des projectiles, les caractéristiques des pointes et de leur emmanchement, la distance et l'angle de tir, etc.

\section{Rappel du protocole expérimental (Pétillon, 2005, 2006)}

Le protocole expérimental a été élaboré en regard du contexte archéologique de référence : le Magdalénien supérieur d'Isturitz (Pyrénées-Atlantiques). Nous avons ainsi fabriqué avec des outils en silex 96 pointes en bois de renne, dont 78 pointes à base fourchue - ce type de base étant largement dominant dans l'ensemble archéologique. Nous les avons emmanchées sur des hampes de projectile en pin, l'une des seules essences que nous pouvions supposer accessibles dans l'environnement du site d'après les quelques données palynologiques disponibles (LeroiGourhan, 1959). La moitié des pointes était fixée sur des flèches destinées à être tirées à l'arc, l'autre moitié équipant des sagaies destinées à être lancées au propulseur. L'utilisation de ces deux modes de propulsion se justifiait du fait que nous n'avions aucune certitude a priori 
quant au(x) type(s) d'arme utilisé(s) au Magdalénien supérieur (Pétillon, 2006). Tous les tirs ont été effectués par des tireurs expérimentés : P. Cattelain (arc, 2003 et 2004 ; propulseur, 2004), P. Chauvaux (propulseur, 2003 et 2004), E. Demoulin et F. Rivère (propulseur, 2004).

En ce qui concerne le choix de la cible, nous n'avons pas pu nous procurer de rennes, gibier dominant dans l'ensemble archéologique (Letourneux in Pétillon et al., sous presse). Les tirs de 2003 ont donc été effectués sur deux carcasses de veaux mâles âgés de quelques semaines et pesant environ $30 \mathrm{~kg}$, et les tirs de 2004 sur 2 daines adultes de $40 \mathrm{~kg}$ environ. Dans les deux cas, les animaux ont été abattus environ une heure avant le début de l'expérimentation, et suspendus à un portique en bois situé à une dizaine de mètres des tireurs (fig. 1). Les animaux étaient orientés le flanc tourné vers les tireurs, de sorte qu'un seul côté était exposé aux tirs. Pour pouvoir distinguer les impacts de flèches des impacts de sagaies, chaque animal été visé avec une seule arme - on a donc au total un veau tiré à l'arc, un veau tiré au propulseur, une daine tirée à l'arc et une daine tirée au propulseur.

Chaque projectile était tiré plusieurs fois, jusqu'à endommagement de la pointe, de l'emmanchement ou de la hampe. Sur les 618 tirs effectués, 455 ont touché la cible. Chaque impact a été systématiquement photographié et a fait l'objet d'une fiche d'enregistrement (fig. 2). A l'issue de chaque journée de tirs, l'animal utilisé a été dépecé sur place, avec des outils en silex. Les ossements décharnés ont été emballés sous vide, avant que leur nettoyage ne soit achevé la semaine suivante au Paléoscope des Mureaux (Yvelines). Ils ont alors été mis à bouillir pour détacher les résidus organiques (restes de chair, nerfs, cartilages, etc.), nettoyés avec les mêmes outils en silex, puis séchés à l'air libre et stockés individuellement.

\section{Nomenclature des traces d'impacts}

A notre connaissance, la seule nomenclature disponible pour les traces d'impacts de projectiles sur les os est celle établie par P. Morel (ibid.), qui distingue cinq catégories :

- les raclements, atteintes superficielles de l'os ;

- les éclatements ou dislocations, où l'os est fragmenté ;

- les fissurations, où l'os reste entier, quoique fendu ;

- les implantations, insertions d'apex brisés lors de l'impact;

- les perforations, empreintes de pointes extraites de l'os.

Nous avons repris cette terminologie, mais nos observations nous ont amenés à hiérarchiser de façon différente les critères de classement. Nous avons ainsi retenu trois catégories de traces, distinguées selon leur dynamique de formation :

- l'encoche (terme que nous avons préféré à "raclement"), qui se produit lorsqu'une pointe frôle un os, arrachant une petite quantité de matière le long d'un de ses bords (fig. 3) ;

- le percement, qui résulte de la pénétration de la pointe à l'intérieur de l'os, sans que celle-ci ressorte de l'autre côté (fig. 4);

- le transpercement, qui correspond au cas où la pointe traverse l'os de part en part (fig. 5).

A ces stigmates peuvent s'ajouter deux phénomènes secondaires, surtout associés aux percements et transpercements :

- l'incrustation de la pointe à l'intérieur de la carcasse. Certaines pointes coincées dans l'animal ont pu être dégagées lors du nettoyage et du décharnement de la carcasse ; mais d'autres sont littéralement restées incrustées dans l'os et, même après traitement et séchage de ces derniers, il s'est avéré impossible de dégager les pointes sans les endommager ni détruire l'os (fig. 6) ; 
- les fissurations, qui se propagent à partir du point d'impact en suivant l'axe des fibres osseuses. Lorsque l'os est de petite taille, la fissuration peut aboutir à son éclatement en plusieurs morceaux (fig. 7).

\section{Résultats}

Parmi les 455 tirs ayant touché la cible, beaucoup ont pris en écharpe la peau de l'animal ou frappé dans les muscles ou les viscères, sans atteindre d'os. Au total, nous avons dénombré 127 traces d'impacts sur les ossements des quatre carcasses (tabl. 1). Précisons de plus qu'en 2004, le cumul de très nombreux impacts dans les côtes a abouti à l'éclatement de plusieurs d'entre elles en nombreux fragments; nous avons exclu de nos décomptes ces portions disloquées, puisqu'elles correspondaient à un «acharnement» des tireurs, qui n'a rien à voir avec une situation de chasse réelle. Malgré ces exclusions, on voit que, toutes expérimentations confondues, plus de $70 \%$ des traces se concentrent dans une zone correspondant aux scapulas, au thorax et aux vertèbres lombaires - ceci reflétant simplement la consigne donnée aux tireurs, qui était de viser les zones vitales de l'animal à la hauteur du cœur et des poumons.

\subsection{Nature des traces / degré d'ossification de l'élément anatomique touché}

Quel que soit l'animal touché, les percements sont toujours les stigmates les plus rares, et les transpercements sont bien représentés (tabl. 2). Le fait que les encoches sont plus fréquentes sur le squelette des daines que sur celui des veaux s'explique probablement par le problème des dislocations de côtes, qui ont certainement abouti à éliminer du décompte un assez grand nombre de transpercements.

Cependant, pour certains types d'os et malgré un effectif relativement réduit, une différence semble apparaître entre les deux espèces (tabl. 3). Ainsi, sur les os longs des veaux, les percements et transpercements sont fréquents et presque exclusifs (huit traces sur neuf), tandis que sur les os longs des daines, les encoches sont majoritaires (trois traces sur cinq). Le degré de minéralisation des os, et dans une moindre mesure leur morphologie, offrent à cette situation une explication tout à fait plausible. Les os immatures des veaux, aux diaphyses plus larges et plus massives, ont facilement laissé pénétrer les pointes, tandis que ceux des daines, à la fois plus minces, plus arrondis et plus solides, ont montré une grande résistance aux impacts. Dans la majorité des cas en effet, les projectiles ayant touché ces os semblent avoir " glissé » sur les diaphyses cylindriques et les reliefs arrondis des extrémités, n'occasionnant à l'os que des dégâts mineurs, voire ne laissant aucune trace macroscopique... Cela alors que les projectiles eux-mêmes subissaient presque systématiquement d'importants dommages (Pétillon, 2006).

L'incrustation de la pointe dans l'os dépend également du degré de minéralisation de ce dernier. Les incrustations sont en effet relativement fréquentes sur les veaux, et pratiquement absentes sur les carcasses de daines (tabl. 4). Le squelette immature des veaux a agi comme un véritable "piège à pointes ", les fibres de l'os se resserrant autour de la pointe et empêchant son extraction. Ainsi, sur la scapula du veau tiré à l'arc, sept transpercements ont abouti à trois incrustations (fig. 8) ; tandis que sur la scapula de la daine tirée au propulseur, les cinq transpercements ne se sont accompagnés d'aucune incrustation, mais ont provoqué une fissuration et un éclatement de l'aile (fig. 9) - alors que la scapula de veau, plus « élastique », a conservé son intégrité.

En revanche, les fissurations (tabl. 5) accompagnent fréquemment les percements et les transpercements et ce quel que soit le degré de minéralisation de l'os (l'absence de 
perforations avec fissuration sur les ossements de daines n'est pas significatif vu la faiblesse de l'effectif).

\subsection{Nature des traces / type d'os}

Lorsqu'on met en regard la nature de l'os touché et celle de la trace observée, deux groupes se dessinent (tabl. 6) : d'une part la colonne vertébrale, la cage thoracique et le coxal pour lesquels les encoches sont les dommages les plus fréquents, et d'autre part la scapula et les os longs, pour lesquels les transpercements sont majoritaires. Rappelons toutefois que la plupart des transpercements sur os longs ont été observés sur les squelettes de veau; or nous avons vu que la fréquence de ces traces sur ces os s'expliquait en grande partie par leur faible degré de minéralisation. Si donc on écarte les os longs, on constate que le contraste principal oppose la scapula aux autres os : la scapula est étroitement associée aux transpercements, ce qui n'a rien de surprenant vu sa forme large et mince et sa position presque « en bouclier » sur le corps de l'animal. Même les tirs qui, traversant le corps de l'animal, frappent la face interne de la scapula opposée aboutissent à un transpercement : le cas s'est produit deux fois en 2003 et deux fois en 2004 (toujours au propulseur).

La forme de l'os - étroite et mince - explique sans doute aussi la distribution particulière des phénomènes secondaires sur les côtes (tabl. 7 et 8). Ces dernières, plus que tous les autres éléments anatomiques, présentent fréquemment des fissurations alors que les incrustations sont absentes.

Précisons enfin que les effectifs réduits enregistrés pour l'ensemble céphalique ne permettent pas de discussion détaillée.

\subsection{Nature des traces / arme utilisée}

A type d'os et à cible comparable, arc et propulseur laissent des stigmates très similaires tant dans la nature des traces que dans les phénomènes secondaires associés (tabl. 9 à 11). Tout au plus peut-on relever que, sur les daines, les tirs à l'arc touchant la cage thoracique et la colonne vertébrale ont provoqué en grande majorité des encoches, tandis qu'au propulseur la répartition est plus équilibrée, l'ensemble "percements + transpercements 》 étant même légèrement majoritaire. A titre d'hypothèse, nous suggérons que, le squelette des daines étant très résistant et les flèches étant des projectiles beaucoup plus légers que les sagaies, les impacts des tirs à l'arc ont peut-être été plus souvent déviés par la face latérale de l'os, entraînant une raréfaction des percements et transpercements. Mais cela n'est que l'une des explications possibles.

\section{Application archéologique}

Les stigmates que nous avons présentés sont relativement faciles à repérer. Par ailleurs, ils peuvent difficilement être confondus avec des traces laissées par les carnivores (Binford, 1981; Blumenschine 1986, 1995 ; Blumenschine et Marean, 1993 ; Haynes, 1983), ou avec les traces d'une percussion lancée destinée par exemple à la récupération de la moelle (Blumenschine, 1995 ; Blumenschine et Selvaggio, 1988, 1991 ; Lyman, 1994, p. 326). On pourrait donc s'attendre à ce que ces stigmates d'impacts de projectiles présentent une bonne visibilité archéologique. Or, comme plusieurs auteurs l'ont souligné (Morel, 1993 ; Stodiek, 1993), ce type de trace est rarement signalé sur les ossements fossiles. De fait, si l'on excepte l'inventaire systématique effectué par N. Noe-Nygaard dans les sites mésolithiques danois (Noe-Nygaard, 1974) et l'étude exhaustive des traces de projectile menée par B. Bratlund sur la faune des niveaux hambourgiens et ahrensbourgiens de Stellmoor et Meiendorf (Bratlund, 
1990), nous n'avons recensé que 12 traces d'impacts d'armes de chasse publiées pour l'ensemble du Paléolithique supérieur et du Mésolithique européens (Pétillon et Letourneux, 2003-2004, revu et complété in Letourneux et Pétillon, sous presse).

P. Morel a déjà suggéré plusieurs explications pour rendre compte de ce faible potentiel de préservation et d'identification; la première étant que, dans une chasse réelle, «les impacts sur l'os devraient être minoritaires parce qu'involontaires » (Morel, 1993, p. 56). Nous avons cependant pu vérifier expérimentalement que, pour un tireur visant les parties vitales de l'animal (zone du cœur et des poumons), une légère déviation suffit pour que le projectile vienne frapper une côte, une vertèbre, la scapula, voire la partie proximale de l'humérus. Cet argument à lui seul ne nous semble donc pas suffisant pour rendre compte de la rareté des traces archéologiques.

P. Morel avance cependant un second argument, soulignant que les os les plus susceptibles de porter des traces d'impacts - vertèbres, côtes, partie plane de la scapula - sont précisément parmi ceux dont la préservation est la plus médiocre : leur morphologie les expose à la fragmentation, et surtout, la faible densité de certaines de leurs portions les rend très vulnérables aux altérations post-dépositionnelles (conservation différentielle : Lyman, 1994 ; Lam et Pearson, 2003, 2004 ; Lam et al., 1998, 1999). Ces os figurent également parmi les éléments les plus souvent consommés par les carnivores (Blumenschine et Marean, 1993) et/ou par les hommes. Ces facteurs expliquent probablement en grande partie la rareté des traces d'impacts sur les vestiges de faune archéologiques. Ils expliquent peut-être également la fréquence plus grande des traces d'impacts dans les sites de tourbières du Paléolithique final et du Mésolithique d'Europe du nord: la préservation de la matière osseuse y est optimale, l'intervention des carnivores souvent minime, et les activités de boucherie parfois totalement absentes (découvertes d'animaux isolés : cf. Noe-Nygaard, 1974).

Malgré ces restrictions, il nous semble cependant que la rareté des traces de projectiles identifiées sur les vestiges de faune archéologiques relève également d'une méconnaissance de ces stigmates qui, souvent, ne sont pas recherchés. L'objectif du référentiel expérimental présenté ici est de remédier en partie à cette méconnaissance et de faciliter l'identification future de traces similaires.

Les stigmates expérimentaux que nous avons présentés ne sauraient cependant constituer un « catalogue universel » de traces d'impacts de projectiles. La mise en regard des deux séances de tir montre à quel point il est difficile de tirer des conclusions de portée générale à partir de résultats isolés. Ainsi, ici, c'est parce qu'il y a eu une seconde expérimentation impliquant un animal d'âge et d'espèce différents que nous avons pu prendre pleinement conscience de l'importance des facteurs intrinsèques dans la formation des traces d'impacts. Les résultats d'une expérimentation sont conditionnés par l'ensemble des éléments du protocole expérimental - y compris ceux dont on n'avait pas soupçonné a priori l'importance - et doivent être interprétés dans les limites de celui-ci. Ce constat souligne la nécessité de poursuivre ce type d'approche, en multipliant les expérimentations avec des protocoles diversifiés, afin de rendre compte de cette variabilité.

\section{Remerciements}

Nous adressons nos plus sincères remerciements à toutes les personnes ayant participé aux expérimentations de 2003 et 2004, en particulier Pierre Cattelain et Claire Bellier. Ces expérimentations ont pu avoir lieu grâce au soutien financier et logistique du CEDARC / Musée du Malgré-Tout, de l'UMR 7041 ArScAn (département d'ethnologie préhistorique), de l'université Paris I Panthéon-Sorbonne et du Paléoscope des Mureaux. Nous remercions 
également Sandrine Costamagno, Philippe Fosse et Frédéric Laudet de nous avoir conviés à la table ronde qu'ils organisaient. Nous sommes reconnaissants à Sandrine Costamagno et Véronique Laroulandie qui, par leurs remarques, critiques et commentaires, ont largement contribué à améliorer une précédente version de ce texte. Cette contribution a été rédigée alors que l'un d'entre nous (J.M.P.) bénéficiait d'un contrat de recherche de la Région Ile-deFrance, tandis que l'autre (C.L.) bénéficiait d'un contrat EIF Marie-Curie au département d'évolution humaine du MPI-EVA de Leipzig.

\section{Bibliographie}

Binford R.L. (1981). Bones : Ancient men and modern myths. Academic Press, New York, $320 \mathrm{p}$.

Blumenschine J.R. (1986). Early hominid scavenging opportunities; implications of carcass availability in the Serengeti and Ngororongoro ecosystems. British Archaeological Reports International Series 283, Oxford, 163 p.

Blumenschine J.R. (1995). Percussion marks, tooth marks, and experimental determinations of the timing of hominid and carnivore access to long bones at FLK Zinjanthropus, Olduvai Gorge, Tanzania. Journal of Human Evolution, 29 : 21-51.

Blumenschine J.R., Marean C.W. (1993). A Carnivore's view of archaeological bone assemblages. In J. Hudson (Ed.), From Bones to Behavior. Center for Archaeological Investigations - Southern Illinois University, Carbonedale : 273-300.

Blumenschine J.R., Selvaggio M.M. (1988). Percussion marks on bone surfaces as a new diagnostic of hominid behavior. Nature, $333:$ 763-765.

Blumenschine J.R., Selvaggio M.M. (1991). On the marks of marrow processing by hammerstones and hyenas: Their anatomical pattering and archaeological implications. In J.D. Clark (Ed.), Cultural beginnings: Approaches to understanding early hominid life-ways in the african savana. Dr Rudolf Habelt GMBH, Bonn : 17-32.

Bratlund B. (1990). Rentierjagd im Spätglazial. Eine Untersuchung der Jagdfrakturen an Rentierknochen von Meiendorf und Stellmoor. Offa, 47 : 7-34.

Haynes G. (1983). A guide for differentiating mammalian carnivore taxa responsible for gnaw damage to herbivore limb bones. Paleobiology, $9: 164-172$.

Lam Y.M., Chen X., Marean C.W., Frey C. (1998). Bone density and long bone representation in archaeological faunas: comparing results from $\mathrm{CT}$ and photon densitometry. Journal of Archaeological Science, $25: 559-570$.

Lam Y.M., Chen X., Pearson O.M. (1999). Intertaxonomic variability in patterns of bone density and the differential representation of bovid, cervid, and equid elements in the archaeological record. American Antiquity, 64 : 343-362.

Lam Y.M., Pearson O.M. (2003). Bone density studies and the interpretation if the faunal record. Evolutionary Anthropology, 14 : 99-108.

Lam Y.M., Pearson O.M. (2004). The fallibility of bone density values and their use in archaeological record. Journal of Taphonomy, 2 : 99-115.

Leroi-Gourhan Arl. (1959). Résultats de l'analyse pollinique de la grotte d'Isturitz. Bulletin de la Société préhistorique française, 56 (9-10) : 619-624. 
Letourneux C., Pétillon J.M. (sous presse). Hunting lesions caused by osseous projectile points: experimental results and implications for the Upper Paleolithic. Journal of Archaeological Science.

Lyman R.L. (1994). Vertebrate Taphonomy. Cambridge University Press, Cambridge, 524 p.

Morel P. (1993). Impacts de projectiles sur le gibier : quelques éléments d'une approche expérimentale. In P. Anderson, S. Beyries, M. Otte, H. Plisson (Eds.), Traces et fonction : les gestes retrouvés. Editions de l'Université de Liège (ERAUL 50), Liège : 55-57.

Morel P. (2000). Impacts de chasse et archéozoologie : quelques observations expérimentales. In C. Bellier, P. Cattelain, M. Otte (Eds.), La chasse dans la Préhistoire. Société royale belge d'Anthropologie et de Préhistoire (Anthropologie et Préhistoire 111) / Editions de l'Université de Liège (ERAUL 51) / Editions du CEDARC (Artefacts 8), Bruxelles : 54-59.

Noe-Nygaard N. (1974). Mesolithic hunting in Denmark illustrated by bone injuries caused by human weapons. Journal of Archaeological Science, $1: 217-248$.

Pétillon J.M. (2005). Tir expérimental de pointes à base fourchue en bois de renne. In: V. Dujardin (Ed.), Industrie osseuse et parures du Solutréen au Magdalénien en Europe. Société préhistorique française (mémoires 34), Paris : 243-256.

Pétillon J.M. (2006). Des Magdaléniens en armes. Technologie des armatures de projectile en bois de Cervidé du Magdalénien supérieur de la grotte d'Isturitz (Pyrénées-Atlantiques). Editions du CEDARC (Artefacts 10), Treignes.

Pétillon J.M., Letourneux C. (2003-2004). Au retour de la chasse... Observations expérimentales concernant les impacts sur le gibier, la récupération et la maintenance des projectiles dans le Magdalénien supérieur d'Isturitz (Pyrénées-Atlantiques). In A. Averbouh, M. Christensen (Eds.), Transformation et utilisation préhistoriques des matières osseuses : actualité des recherches universitaires en France 2000-2004. Préhistoire et Anthropologie méditerranéennes, $12: 173-188,13: 133$.

Pétillon J.M., Letourneux C., Laroulandie V. (sous presse). Archéozoologie des collections anciennes : le cas de la faune du Magdalénien supérieur d'Isturitz. In C. Normand (Ed.), Les recherches archéologiques dans les grottes d'Isturitz et d'Oxocelhaya de 1912 à nos jours : une synthèse des résultats. Actes de la table ronde du cinquantenaire du classement comme Monument Historique des grottes d'Isturitz et d'Oxocelhaya, Hasparren, 14-15 novembre 2003. Paléo hors-série.

Stodiek U. (1991). Erste Ergebnisse experimenteller Untersuchungen von Gewiehgeschoßspitzen des Magdalénien, Experimentelle Archäologie. Bilanz 1991, Archäologische Mitteilungen aus Nordwestdeutschland Beiheft 6: 245-256.

Stodiek U. (1993). Zur Technologie der jungpaläolithischen Speerschleuder. Eine Studie auf der Basis archäologischer, ethnologischer und experimenteller Erkenntnisse. Archaeologia Venatoria (Tübinger Monographien zur Urgeschichte 9), Tübingen.

Stodiek U. (2000). Preliminary results of an experimental investigation of Magdalenian antler points. In C. Bellier, P. Cattelain, M. Otte (Eds.), La chasse dans la Préhistoire. Société royale belge d'Anthropologie et de Préhistoire (Anthropologie et Préhistoire 111)/Editions de l'Université de Liège (ERAUL 51) / Editions du CEDARC (Artefacts 8), Bruxelles : 70-78. 
Fig. 1 : vue générale du pas de tir pendant l'expérimentation 2004. On voit à droite l'animal cible suspendu au portique en bois. Pierre Cattelain, entouré en noir, vient de tirer au propulseur (cliché D. Henry-Gambier).

Fig. 1 : general view of the shooting field during the 2004 experiments. The target animal suspended to a wooden bracket can be seen on the right. Pierre Cattelain (black circle) has just thrown a spear (photograph by D. Henry-Gambier).

Fig. 2 : expérimentation 2004. Fiche utilisée pour l'enregistrement de chaque tir. Fig. 2 : 2004 experiment. Sheet used for the recording of each individual shot.

Fig. 3 : exemples d'encoches. a : sur une côte (veau, tir à l'arc). b : sur un radio-ulna (daine, tir au propulseur).

Fig. 3 : notches. a : rib, ox calf, bow shot. $\mathrm{b}$ : radio-ulna, fallow deer, spearthrower shot.

Fig. 4 : percement d'une extrémité distale d'humérus, avec fissuration et incrustation (veau, tir à 1 'arc).

Fig. 4 : puncture with crack and embedding. Distal extremity of humerus, ox calf, bow shot.

Fig. 5 : exemples de transpercements. a : sur un radio-ulna, avec fissuration et incrustation (veau, tir au propulseur). $\mathrm{b}$ : sur une côte, avec fissuration (daine, tir au propulseur).

Fig. 5 : perforations. a : with crack and embedding; radio-ulna, ox calf, spearthrower shot. $\mathrm{b}$ : with crack; rib, fallow deer, spearthrower shot.

Fig. 6 : fissurations d'un humérus suite à deux transpercements, dont un avec incrustation (veau, tir à l'arc).

Fig. 6: two perforations with cracks, one of them with additional embedding of the point (humerus, ox calf, bow shot).

Fig. 7 : transpercement de l'extrémité proximale d'un tibia, avec fissuration. L'os a en fait subi deux impacts très rapprochés (daine, tir à l'arc).

Fig. 7 : perforations with cracks. The bone actually suffered two very close impacts (proximal extremity of tibia, fallow deer, bow shots).

Fig. 8 : scapula ayant subi sept transpercements, dont trois avec incrustation (veau, tir à l'arc). Fig. 8 : scapula with seven perforations, three of them with embedding of the point (ox calf, bow shots).

Fig. 9 : scapula ayant subi cinq transpercements avec fissuration (1 sur l'épine à droite, 4 sur l'aile à gauche). Les 4 impacts sur l'aile ont entraîné son éclatement (daine, tir au propulseur). Fig. 9 : scapula with five perforations, all of them with cracks. The four cracks on the distal part splintered the bone (fallow deer, spearthrower shots). 


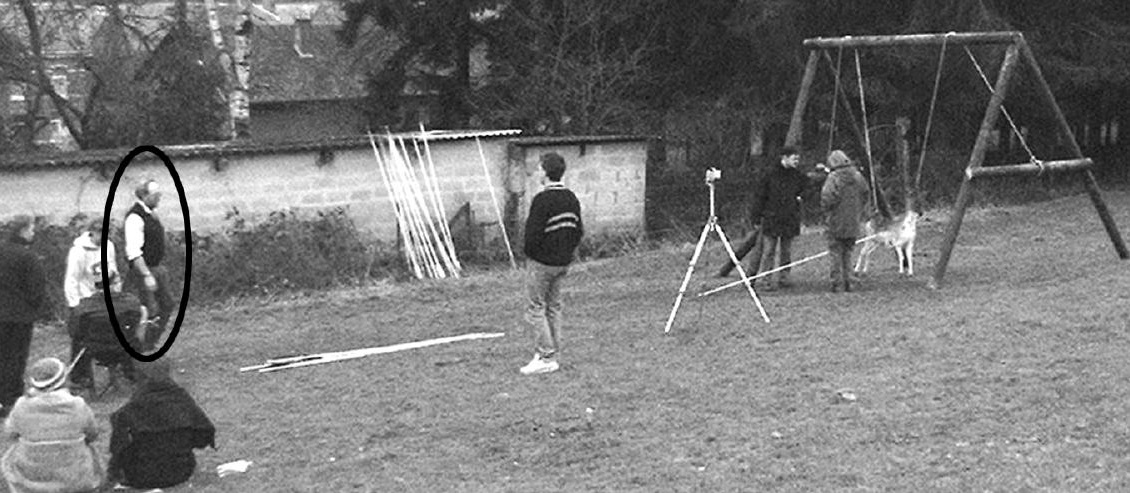




\section{$\square$ TIR AU BUT}

Organe ou os touché :

Longueur projectile restante :

Si projectile a transpercé la cible : $\square$

Extraction du projectile :

$\square$ Sans problème

$\square$ Pointe se démanche à l'extraction et reste dans la cible

$\square$ Pointe fracturée, fragment laissé dans la cible

\section{口 TIR MANQUÉ}

$\square$ Tir manque la cible, atterrit dans le sol

$\square$ Tir "ripé" (glisse sur l'animal et atterrit dans le sol)

$\square$ Tir frappe la charpente en bois

$\square$ Autre :

\section{FRACTURE DE LA POINTE}

$\square$ Fracture mésiale ou distale

$\square$ Fracture d'un fourchon

$\square$ Fracture des deux fourchons

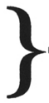

Le fragment distal ou mésio-distal est-il :

$\square$ Récupéré

$\square$ Laissé dans la cible $\square$ Perdu

口 FRACTURE DU FÛT :

Fracture au niveau de l'emmanchement

7 Autre :

\section{REMARQUES}




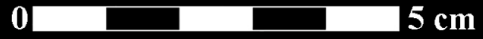



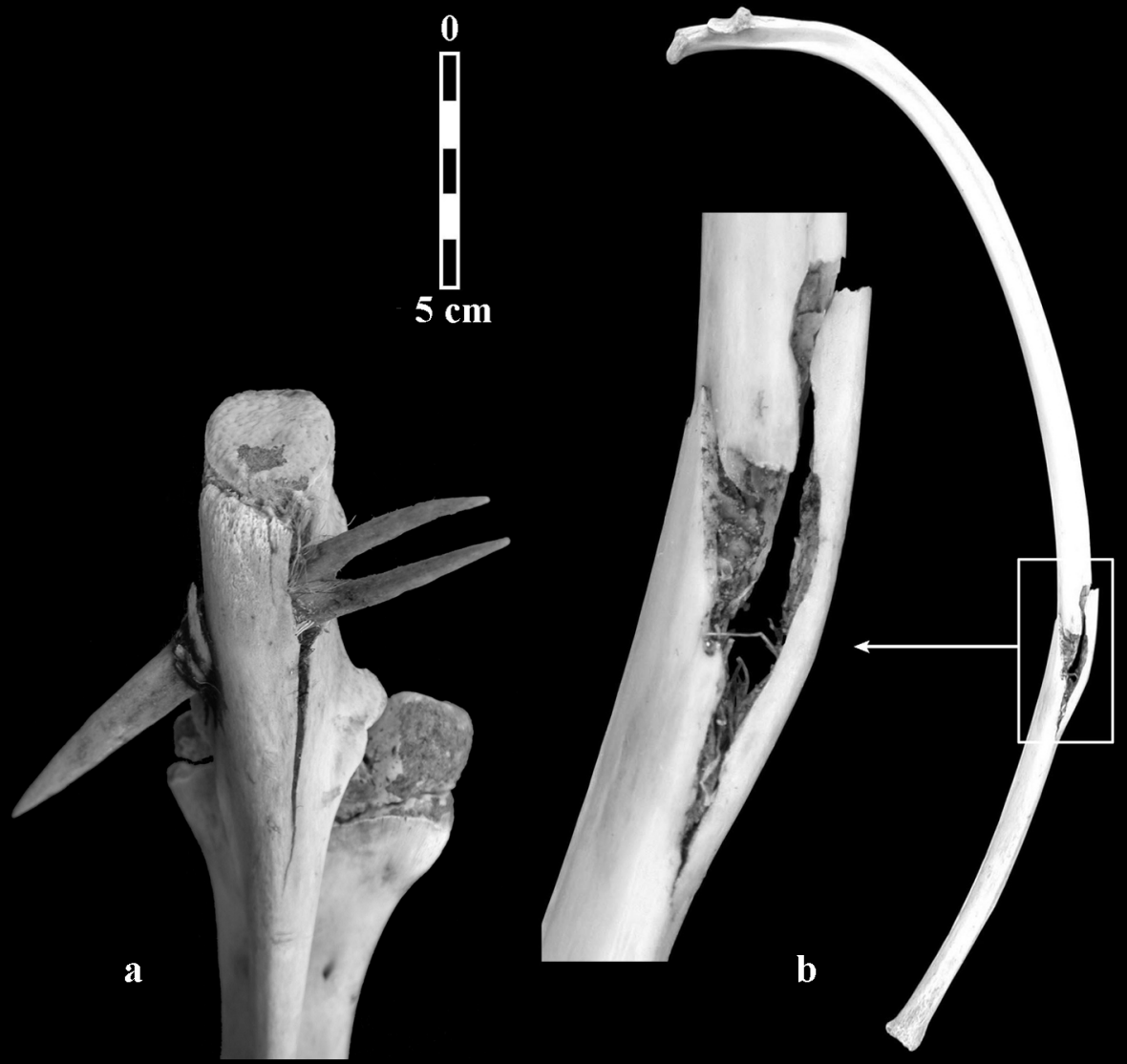


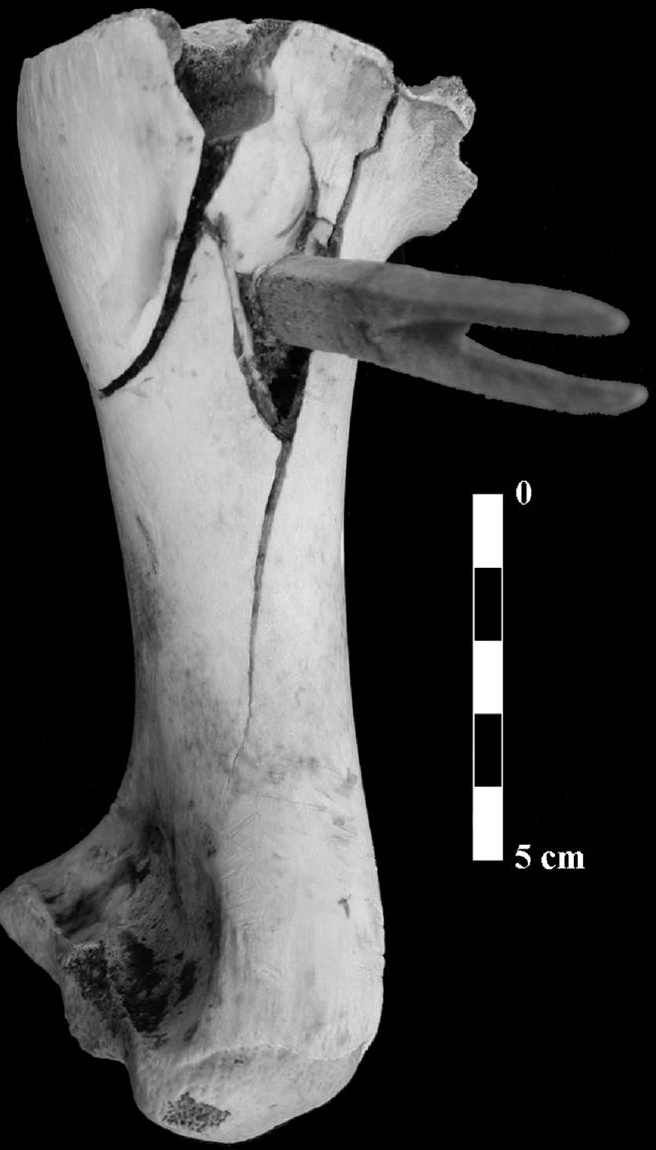




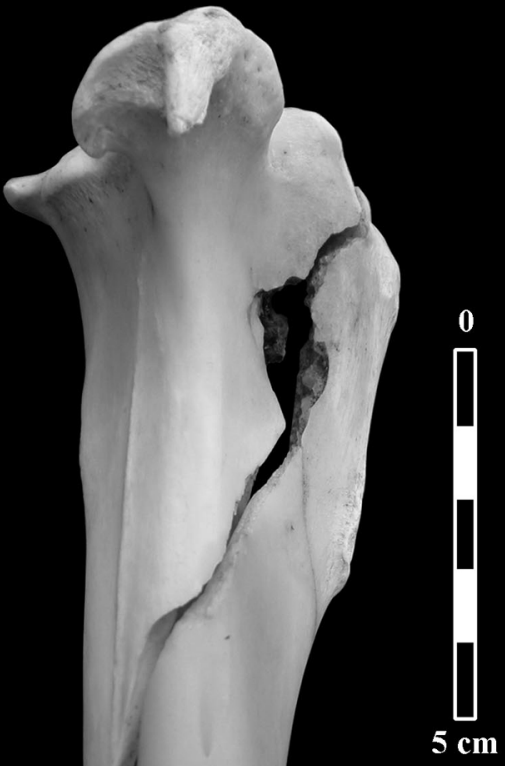



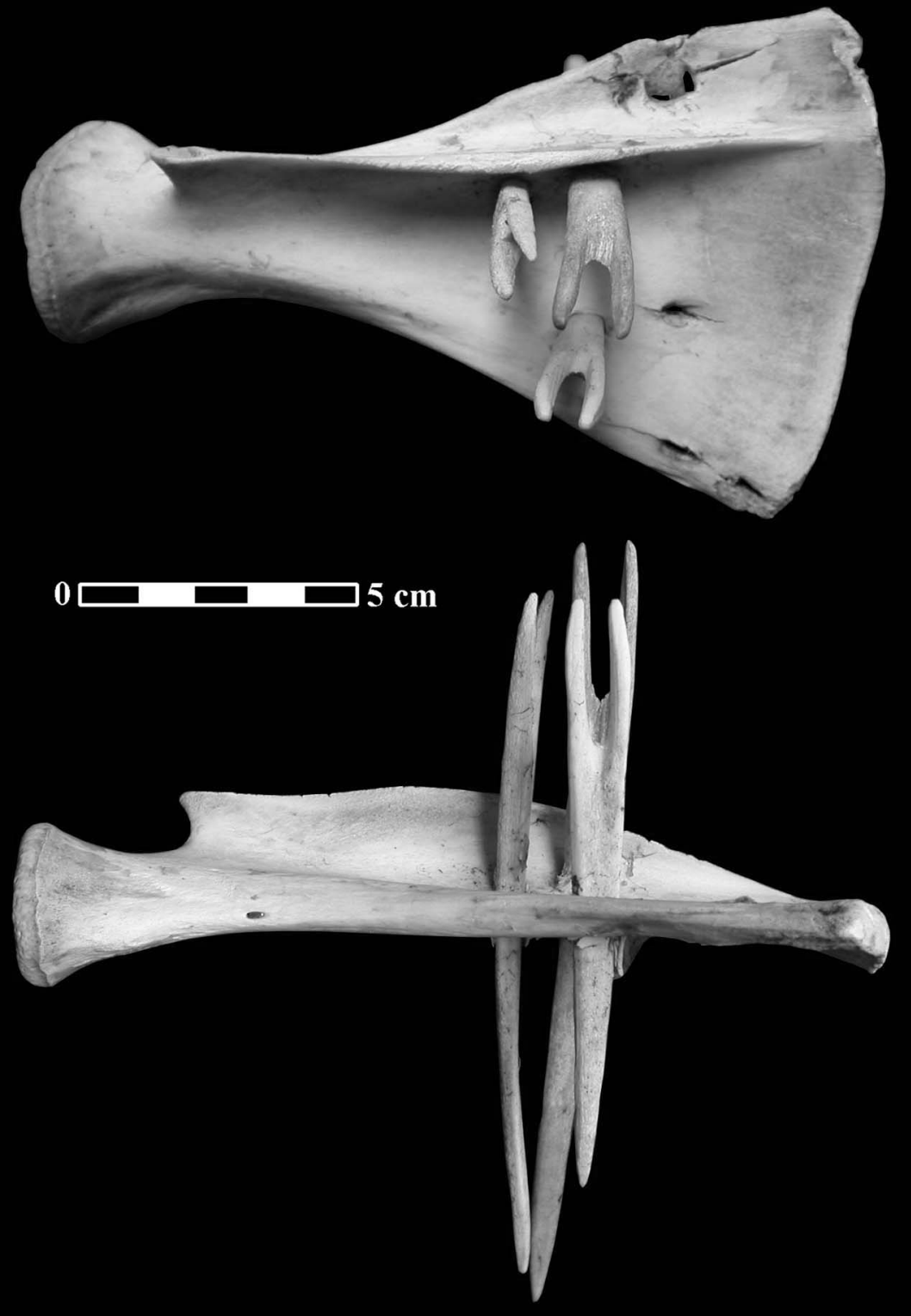


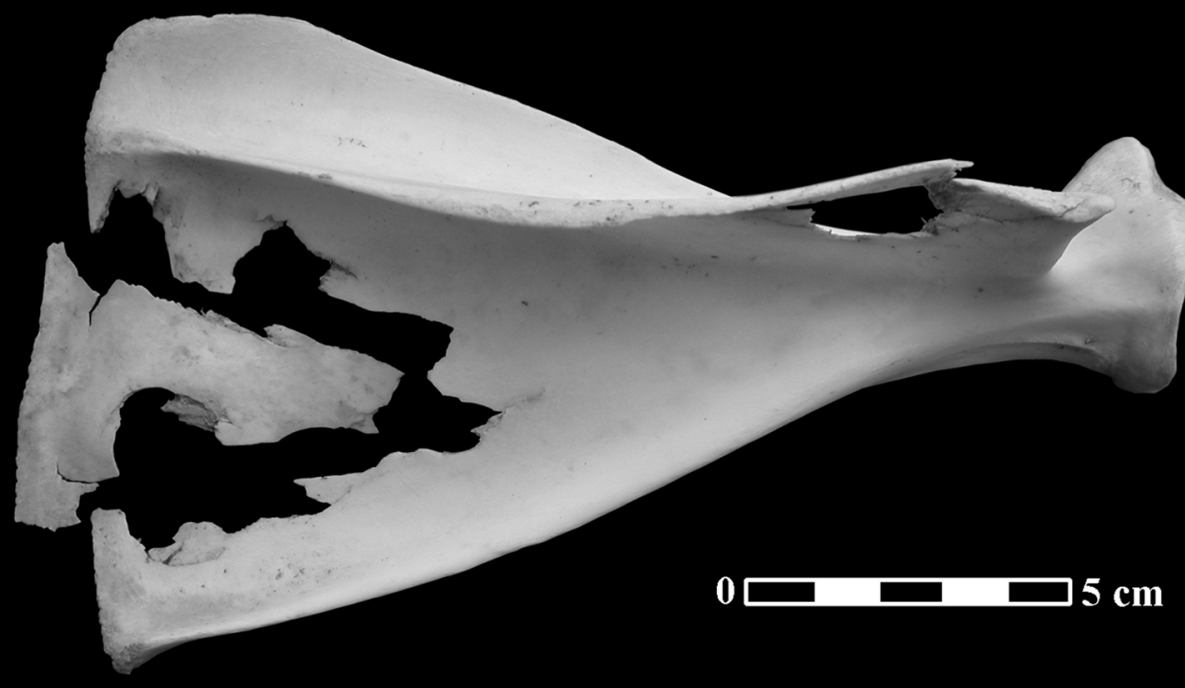




\begin{tabular}{|c|c|c|c|c|c|c|c|c|}
\hline \multirow{2}{*}{ Région anatomique touchée } & \multicolumn{2}{|c|}{ Veaux 2003} & \multicolumn{2}{|c|}{ Daines 2004} & \multicolumn{4}{|c|}{ Total tirs 2003-2004 } \\
\hline & Arc & Propusleur & Arc & Propulseur & Arc & Propulseur & Total & $\%$ Total \\
\hline Tête & - & 1 & - & 1 & - & 2 & 2 & 1,6 \\
\hline V. cervicales & - & 2 & 1 & 9 & 1 & 11 & 12 & 9,4 \\
\hline V. thoraciques et lombaires & 9 & 4 & 5 & 19 & 14 & 23 & 37 & 29,1 \\
\hline Côtes & 7 & 4 & 7 & 10 & 14 & 14 & 28 & 22,0 \\
\hline Sternum & 2 & - & 1 & 2 & 3 & 2 & 5 & 3,9 \\
\hline Scapula & 7 & 4 & 2 & 7 & 9 & 11 & 20 & 15,7 \\
\hline Humérus & 6 & 1 & - & 2 & 6 & 3 & 9 & 7,1 \\
\hline Radio-ulna & 一 & 2 & - & 1 & - & 3 & 3 & 2,4 \\
\hline Coxal & - & 一 & 1 & 4 & 1 & 4 & 5 & 3,9 \\
\hline Sacrum & 一 & 一 & 1 & 3 & 1 & 3 & 4 & 3,1 \\
\hline Tibia & 一 & 一 & 2 & 一 & 2 & 一 & 2 & 1,6 \\
\hline Nombre de traces & 31 & 18 & 20 & 58 & 51 & 76 & 127 & 100,0 \\
\hline
\end{tabular}

Tabl. 1 : distribution anatomique des traces d'impacts observées sur les animaux-cibles en fonction de l'arme utilisée. $V=$ vertèbres.

Tab. 1: skeletal distribution of the different impact traces by species and weapon. $V=$ vertebrae 


\begin{tabular}{|l|c|c|c|c|c|c|c|c|}
\hline \multirow{2}{*}{ Animal-cible } & \multicolumn{2}{|c|}{ Encoches } & \multicolumn{2}{|c|}{ Percements } & \multicolumn{2}{c|}{ Transpercements } & \multicolumn{3}{c|}{ Total des traces } \\
& $\mathrm{N}$ & $\% \mathrm{~N}$ & $\mathrm{~N}$ & $\% \mathrm{~N}$ & $\mathrm{~N}$ & $\% \mathrm{~N}$ & $\mathrm{~N}$ & $\% \mathrm{~N}$ \\
\hline Veaux & 14 & 28,6 & 6 & 12,2 & 29 & 59,2 & 49 & 100,0 \\
Daines & 38 & 48,7 & 3 & 3,8 & 37 & 47,4 & 78 & 100,0 \\
\hline Total & 52 & 40,9 & 9 & 7,1 & 66 & 52,0 & 127 & 100,0 \\
\hline
\end{tabular}

Tabl. 2 : distribution typologique des traces d'impacts sur chaque animal-cible. Tab. 2: impact traces by species. 


\begin{tabular}{|c|c|c|c|c|c|c|}
\hline \multirow{2}{*}{ Région anatomique touchée } & \multicolumn{3}{|c|}{ Veaux 2003} & \multicolumn{3}{|c|}{ Daines 2004} \\
\hline & Encoches & Percements & Transpercements & Encoches & Percements & Transpercements \\
\hline Tête & - & - & 1 & - & - & 1 \\
\hline V. cervicales & - & - & 2 & 7 & - & 3 \\
\hline V. thoraciques et lombaires & 7 & 1 & 5 & 13 & 1 & 10 \\
\hline Sacrum & - & - & - & - & - & 4 \\
\hline Sternum & $\overline{-}$ & 2 & $\overline{-}$ & 2 & - & 1 \\
\hline Côtes & 6 & - & 5 & 10 & 2 & 5 \\
\hline Coxal & - & - & - & 3 & - & 2 \\
\hline Scapula & - & 1 & 10 & - & - & 9 \\
\hline Humérus & 1 & 2 & 4 & 2 & - & - \\
\hline Radio-ulna & - & - & 2 & 1 & - & - \\
\hline Tibia & - & - & - & - & - & 2 \\
\hline Nombre de traces & 14 & 6 & 29 & 38 & 3 & 37 \\
\hline
\end{tabular}

Tabl. 3 : distribution typologique et anatomique des traces d'impacts sur chaque animal-cible, en fonction de la nature de l'os. $V=$ vertèbres.

Tab. 3: skeletal distribution of the different impact traces by species and bone type. $V=$ vertebrae. 


\begin{tabular}{|l|c|c|c|c|c|c|}
\hline \multirow{2}{*}{ Animal-cible } & \multicolumn{2}{|c|}{ Percements + incrustations } & \multicolumn{2}{c|}{ Transpercements + incrustations } & \multicolumn{2}{c|}{ Total des traces } \\
& $\mathrm{N}$ & \%Fréquence & $\mathrm{N}$ & \%Fréquence & $\mathrm{N}$ & \%Fréquence \\
\hline Veaux & 2 & 33,3 & 10 & 34,5 & 12 & 24,5 \\
Daines & 0 & 0,0 & 1 & 2,7 & 1 & 1,3 \\
\hline Total & 2 & 22,2 & 11 & 16,7 & 13 & 10,2 \\
\hline
\end{tabular}

Tabl. 4 : distribution des incrustations par type de trace sur chaque animal-cible.

Le \%Fréquence correspond au rapport entre le nombre d'incrustations et le nombre total de traces du type donné.

Tab. 4: distribution of embeddings by bone type and species.

$\%$ Frequence is the ratio between the number of embeddings and the total number of each type of trace. 


\begin{tabular}{|c|c|c|c|c|c|c|c|c|}
\hline \multirow{2}{*}{ Animal-cible } & \multicolumn{2}{|c|}{ Encoches + fissurations } & \multicolumn{2}{|c|}{ Percements + fissurations } & \multicolumn{2}{|c|}{ Transpercements + fissurations } & \multicolumn{2}{|c|}{ Total des traces } \\
\hline & $\mathrm{N}$ & \%Fréquence & $\mathrm{N}$ & \%Fréquence & $\mathrm{N}$ & $\%$ Fréquence & $\mathrm{N}$ & \%Fréquence \\
\hline Veaux & 0 & 0,0 & 3 & 50,0 & 17 & 58,6 & 20 & 40,8 \\
\hline Daines & 6 & 15,8 & 0 & 0,0 & 19 & 51,4 & 25 & 32,1 \\
\hline Total & 6 & 11,5 & 3 & 33,3 & 36 & 54,5 & 45 & 35,4 \\
\hline
\end{tabular}

Tabl. 5 : distribution des fissurations par type de trace sur chaque animal-cible.

Le \%Fréquence correspond au rapport entre le nombre de fissurations et le nombre total de traces du type donné.

Tab. 5: distribution of cracks by bone type and species.

$\%$ Frequence is the ratio between the number of cracks and the total number of each type of trace. 


\begin{tabular}{|c|c|c|c|c|c|c|c|c|c|}
\hline & \multicolumn{2}{|c|}{ Encoches } & \multicolumn{2}{|c|}{ Percements } & \multicolumn{2}{|c|}{ Transpercements } & \multicolumn{2}{|c|}{ Total } \\
\hline & & $\mathrm{N}$ & $\% \mathrm{~N}$ & $\mathrm{~N}$ & $\% \mathrm{~N}$ & $\mathrm{~N}$ & $\% \mathrm{~N}$ & $\mathrm{~N}$ & $\% \mathrm{~N}$ \\
\hline \multicolumn{2}{|c|}{$\begin{array}{l}\text { Régions anatomiques touchées } \\
\text { Tôto }\end{array}$} & 0 & 0,0 & 0 & 0,0 & 2 & 100,0 & 2 & 100,0 \\
\hline \multicolumn{2}{|c|}{ Colonne vertébrale } & 27 & 50,9 & 2 & 3,8 & 24 & 45,3 & 53 & 100,0 \\
\hline \multicolumn{2}{|c|}{ Cage thoracique } & 18 & 54,5 & 4 & 12,1 & 11 & 33,3 & 33 & 100,0 \\
\hline \multirow{2}{*}{ Ceintures } & scapula & 0 & 0,0 & 1 & 5,0 & 19 & 95,0 & 20 & 100,0 \\
\hline & coxal & 3 & 60,0 & 0 & 0,0 & 2 & 40,0 & 5 & 100,0 \\
\hline \multicolumn{2}{|l|}{ Os longs } & 4 & 28,6 & 2 & 14,3 & 8 & 57,1 & 14 & 100,0 \\
\hline \multicolumn{2}{|l|}{ Total } & 52 & 40,9 & 9 & 7,1 & 66 & 52,0 & 127 & 100,0 \\
\hline
\end{tabular}

Tabl. 6 : distribution anatomique des traces d'impacts.

Tab. 6: skeletal distribution of impact traces. 


\begin{tabular}{|c|c|c|c|c|c|c|c|c|c|}
\hline \multirow{2}{*}{\multicolumn{2}{|c|}{ Régions anatomiques touchées }} & \multicolumn{2}{|c|}{ Encoches + fissuration } & \multicolumn{2}{|c|}{ Percements + fissuration } & \multicolumn{2}{|c|}{ Transpercements + fissuration } & \multicolumn{2}{|c|}{ Total } \\
\hline & & $\mathrm{N}$ & \%Fréquence & $\mathrm{N}$ & \%Fréquence & $\mathrm{N}$ & réquence & $\mathrm{N}$ & \%Fréquence \\
\hline Tête & & 0 & 0,0 & 0 & 0,0 & 1 & 50,0 & 1 & 50,0 \\
\hline Colonne ve & & 1 & 3,7 & 0 & 0,0 & 9 & 37,5 & 10 & 18,9 \\
\hline Cage thora & & 5 & 27,8 & 1 & 25,0 & 9 & 81,8 & 15 & 45,5 \\
\hline Ceintures & $\begin{array}{l}\text { scapula } \\
\text { coxal }\end{array}$ & $\begin{array}{l}0 \\
0\end{array}$ & $\begin{array}{l}0,0 \\
0,0\end{array}$ & $\begin{array}{l}1 \\
0 \\
\end{array}$ & $\begin{array}{c}100,0 \\
0,0\end{array}$ & $\begin{array}{c}10 \\
0\end{array}$ & $\begin{array}{c}52,6 \\
0,0\end{array}$ & $\begin{array}{c}11 \\
0\end{array}$ & $\begin{array}{c}55,0 \\
0,0\end{array}$ \\
\hline Os longs & & 0 & 0,0 & 1 & 50,0 & 7 & 87,5 & 8 & 57,1 \\
\hline Total & & 6 & 11,5 & 3 & 33,3 & 36 & 54,5 & 45 & 35,4 \\
\hline
\end{tabular}

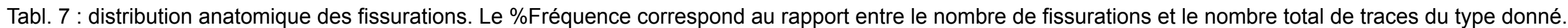
Tab. 7: skeletal distribution of cracks. \%Frequence is the ratio between the number of cracks and the total number of each type of trace. 


\begin{tabular}{|c|c|c|c|c|c|c|c|}
\hline \multirow{2}{*}{\multicolumn{2}{|c|}{ Régions anatomiques touchées }} & \multicolumn{2}{|c|}{ Percements + incrustations } & \multicolumn{2}{|c|}{ Transpercements + incrustations } & \multicolumn{2}{|c|}{ Total } \\
\hline & & $\mathrm{N}$ & $\%$ Fréquence & $\mathrm{N}$ & \%Fréquence & $\mathrm{N}$ & \%Fréquence \\
\hline Tête & & 0 & 0,0 & 0 & 0,0 & 0 & 0,0 \\
\hline Colonne ve & & 0 & 0,0 & 3 & 12,5 & 3 & 5,7 \\
\hline Cage thora & & 0 & 0,0 & 0 & 0,0 & 0 & 0,0 \\
\hline Ceintures & $\begin{array}{l}\text { scapula } \\
\text { coxal }\end{array}$ & $\begin{array}{l}1 \\
0\end{array}$ & $\begin{array}{c}100,0 \\
0,0\end{array}$ & $\begin{array}{l}5 \\
0\end{array}$ & $\begin{array}{c}26,3 \\
0,0\end{array}$ & $\begin{array}{l}6 \\
0\end{array}$ & $\begin{array}{c}30,0 \\
0,0\end{array}$ \\
\hline Os longs & & 1 & 50,0 & 3 & 37,5 & 4 & 28,6 \\
\hline Total & & 2 & 22,2 & 11 & 16,7 & 13 & 10,2 \\
\hline
\end{tabular}

Tabl. 8 : distribution anatomique des incrustations.

Le \%Fréquence correspond au rapport entre le nombre d'incrustations et le nombre total de traces du type donné.

Tab. 8: skeletal distribution of embeddings.

$\%$ Frequence is the ratio between the number of embeddings and the total number of each type of trace. 


\begin{tabular}{|l|c|c|c|c|c|c|c|c|}
\hline \multirow{2}{*}{ Arme utilisée } & \multicolumn{2}{|c|}{ Encoches } & \multicolumn{2}{c|}{ Percements } & \multicolumn{2}{c|}{ Transpercements } & \multicolumn{2}{c|}{ Total } \\
& $\mathrm{N}$ & $\% \mathrm{~N}$ & $\mathrm{~N}$ & $\% \mathrm{~N}$ & $\mathrm{~N}$ & $\% \mathrm{~N}$ & $\mathrm{~N}$ & $\% \mathrm{~N}$ \\
\hline Arc & 22 & 43,1 & 5 & 9,8 & 24 & 47,1 & 51 & 100,0 \\
Propulseur & 30 & 39,5 & 4 & 5,3 & 42 & 55,3 & 76 & 100,0 \\
\hline
\end{tabular}

Tabl. 9 : distribution typologique des traces d'impacts selon l'arme utilisée.

Tab. 9: distribution of impact traces by weapon. 


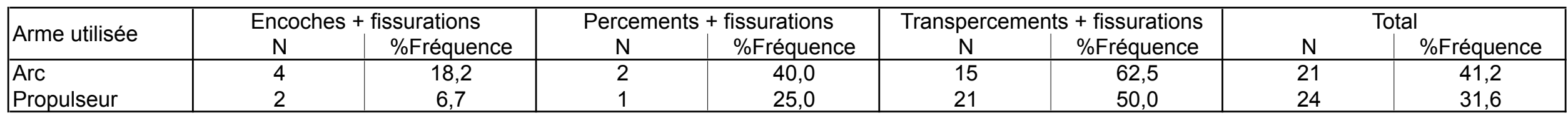

Tabl. 10 : distribution typologique des fissurations selon l'arme utilisée.

Le \%Fréquence correspond au rapport entre le nombre de fissurations et le nombre total de traces du type donné.

Tab. 10: distribution of cracks by weapon.

$\%$ Frequence is the ratio between the number of cracks and the total number of each type of trace. 


\begin{tabular}{|c|c|c|c|c|c|c|}
\hline \multirow{2}{*}{ Arme utilisée } & \multicolumn{2}{|c|}{ Percements + incrustations } & \multicolumn{2}{|c|}{ Transpercements + incrustations } & \multicolumn{2}{|c|}{ Total } \\
\hline & $\mathrm{N}$ & \%Fréquence & $\mathrm{N}$ & $\%$ Fréquence & $\mathrm{N}$ & \%Fréquence \\
\hline & 1 & 20,0 & 4 & 16,7 & 5 & 9,8 \\
\hline Propulseur & 1 & 25,0 & 7 & 16,7 & 8 & 10,5 \\
\hline
\end{tabular}

Tabl. 11 : distribution typologique des incrustations selon l'arme utilisée.

Le \%Fréquence correspond au rapport entre le nombre d'incrustations et le nombre total de traces du type donné.

Tab. 11: distribution of embeddings by weapon.

$\%$ Frequence is the ratio between the number of embeddings and the total number of each type of trace. 